Mon. Not. R. Astron. Soc. 000, 17 (2020) Printed 10 February $2020 \quad$ (MN LATEX style file v2.2)

\title{
Identifying the formation mechanism of redback pulsars
}

\author{
M. A. De Vito ${ }^{1,2 \star}$, O. G. Benvenuto ${ }^{1,2} \dagger$, J. E. Horvath ${ }^{3} \ddagger$, \\ 1 Instituto de Astrofísica de La Plata, IALP, CCT-CONICET-UNLP, Argentina \\ 2 Facultad de Ciencias Astronómicas y Geofísicas, Universidad Nacional de La Plata (UNLP), \\ Paseo del Bosque S/N, B1900FWA, La Plata, Argentina \\ 3 Instituto de Astronomía, Geofísica e Ciências Atmosféricas, Universidade de São Paulo, \\ R. do Matão 1226 (05508-090), Cidade Universitária, São Paulo SP, Brazil
}

February 7, 2020

\begin{abstract}
We analyse the evolution of close binary systems containing a neutron star that lead to the formation of redback pulsars. Recently there has been some debate on the origin of such systems and the formation mechanism of redbacks may still be considered as an open problem. We show that the operation of a strong evaporation mechanism, starting from the moment when the donor star becomes fully convective (or alternatively since the formation of the neutron star by accretion induced collapse), produces systems with donor masses and orbital periods in the range corresponding to redbacks with donors appreciably smaller than their Roche lobes, i.e., they have low filling factors (lower than 0.75). Models of redback pulsars can be constructed assuming the occurrence of irradiation feedback. They have been shown to undergo cyclic mass transfer during the epoch at which they attain donor masses and orbital periods corresponding to redbacks, and stay in quasi-Roche lobe overflow conditions with high filling factors. We show that, if irradiation feedback occurs and radio ejection inhibits further accretion onto the neutron star after the first mass transfer cycle, the redback systems feature high filling factors. We suggest that the filling factor should be considered as a useful tool for discriminating among those redback formation mechanisms. We compare theoretical results with available observations, and conclude that observations tend to favour models with high filling factors.
\end{abstract}

Key words: (stars:) binaries (including multiple): close, (stars:) pulsars: general

\section{INTRODUCTION}

From the study of eclipsing millisecond pulsars (MSPs) belonging to close binary systems (CBSs), evidence has emerged for the existence of two well-separated families of systems: Black Widows (BWs) and Redbacks (RBs) (Roberts 2013). While RBs have circular orbits with orbital periods $0.1 \lesssim P / d \lesssim 1.0$ and companion stars with masses $M_{2}$ in the range $0.1 \lesssim M_{2} / M_{\odot} \lesssim 0.7$, BWs show orbital periods in the same range but substantially lighter companions, with $M_{2} / M_{\odot} \lesssim 0.05$. Besides the range of characteristic masses, it is the state of these companions which differentiates both families. BWs companions are degenerate

\footnotetext{
* Member of the Carrera del Investigador Científico, Consejo Nacional de Investigaciones Científicas y Técnicas (CONICET). Email: adevito@fcaglp.unlp.edu.ar

† Member of the Carrera del Investigador Científico, Comisión de Investigaciones Científicas de la Provincia de Buenos Aires (CIC). Email: obenvenu@fcaglp.unlp.edu.ar $\ddagger$ Email: foton@iag.usp.br
}

stars, while RBs companions are normal stars, with spectral types in a wide range, from $\mathrm{F}$ to $\mathrm{M}$. In addition, for the same effective temperature, donor stars in RBs are brighter than a isolated main sequence star. Therefore, the donor star in a $\mathrm{RB}$ system has a radius greater than an isolated star of the same spectral type. For this reason, it is said that donor stars in RB systems are extended stars.

It is important to understand the mechanisms that give rise to the formation of BWs and RBs. Their very existence challenges the standard treatment Podsiadlowski, Rappaport, \& Pfahl 2002; Benvenuto \& De Vito 2005) of the evolution of CBSs including a neutron star (NS) component. For the case of BWs, a general consensus emerged that they stem from evaporation of the donor star driven by pulsar irradiation (Phinney et al. 1988). On the other hand, the mechanism(s) for the formation of RBs remained controversial. To date there are three mechanisms proposed to account for the existence of RBs: strong evaporation, irradiation feedback, and accretion induced collapse of white dwarfs (WDs). 
A strong evaporation mechanism has been presented in Chen et al. (2013). These authors considered that the pulsar begins to irradiate when the donor star becomes fully convective. At that moment, magnetic braking ceases (it is no longer an angular momentum sink) and the donor detaches from its Roche lobe. Since then on, radio ejection (Burderi et al. 2001) starts to inhibit further accretion onto the NS and forces evaporation. Then, the system evolves to longer orbital periods in the range corresponding to RBs.

Irradiation feedback has been studied by Büning \& Ritter (2004) and applied to RBs by Benvenuto, De Vito, \& Horvath (2014). When the donor star undergoes Roche lobe overflow (RLOF), it starts to transfer mass to the NS. If accretion occurs, the material falling onto the NS leads to the emission of X-ray radiation that illuminates the donor star. If the donor has a thick enough outer convective zone, irradiation may strongly affect its evolution. In many cases this makes the CBS to undergo cyclic mass transfer (Büning \& Ritter 2004). This may occur if radio ejection does not suppress accretion. In between these cycles, mass transfer ceases and the NS may act as a pulsar.

Smedley et al. (2015) proposed that RBs may be formed by accretion induced collapse (AIC) of heavy oxygen, neon and magnesium (ONeMg) WDs. This path to form a RB system contemplates a binary system initially composed of a star of $8-11 M_{\odot}$ and a companion of $\approx 1 M_{\odot}$. After the RLOF of the more massive star, a common envelope stage and envelope ejection, the system results in a ONeMg WD (that initially was the more massive star of the pair) and its companion, that will fill its Roche lobe and start transferring matter to the WD. When the WD reaches the limit mass value of $1.37 M_{\odot}$, it undergoes accretion induced collapse to form a NS. Eventually the system becomes detached and since then on, it is considered to follow an evolution similar to that studied by Chen et al. (2013).

Still, there is room for another formation mechanism: we suggest that if irradiation feedback forces an early detachment from its Roche lobe and mass transfer stops, then pulsar spin-down irradiation may start. If such irradiation inhibits further accretion onto the NS, it is possible to form $\mathrm{RBs}$ even with low evaporation rates. In this scenario it is not necessary to wait for the donor to achieve a mass low enough to become fully convective. Thus, much more massive donors may undergo evaporation and are candidates to become RBs.

Following Boffin et al. (2014), let us define the filling factor as $F F=R_{2} / R_{\mathrm{RL}}$ where $R_{2}$ and $R_{\mathrm{RL}}$ are the radius of the donor star and its corresponding Roche lobe Eggleton 1983), respectively. It will be shown below that the mechanisms that invoke strong evaporation generally lead to the occurrence of donor stars with sizes much smaller than those of their associated Roche lobes. On the contrary, the mechanisms that involve irradiation feedback give rise to donor stars in the quasi-RLOF state, with their lobes almost fully filled. It is the goal of this paper to explore if filling factors may help us to discriminate which is the most probable formation mechanism of RB pulsars.

The remainder of this paper is organized as follows. In Section 2 we describe the treatment of irradiation feedback and evaporation we shall consider in the numerical calculations presented in Section 3. In Section 4 we present the observational data currently available for RB systems and compare it with theoretical calculations. Finally, in Section 5 we discuss the relevance of our results and give some concluding remarks.

\section{CONSIDERING IRRADIATION FEEDBACK AND EVAPORATION}

The calculations to be presented below have been performed with our binary stellar evolution code, described in Benvenuto \& De Vito (2003) and Benvenuto, De Vito, \& Horvath (2014). We shall make a brief summary of the treatment of irradiation feedback and evaporation included in it.

In order to consider irradiation feedback, we assume that the NS acts as a point source releasing an accretion luminosity $L_{\text {acc }}=G M_{1} \dot{M}_{1} / R_{1}$, where $M_{1}, \dot{M}_{1}$, and $R_{1}$ are the mass, accretion rate and radius of the NS, respectively. For isotropic emission, the energy flux incident on the donor star that effectively participates in the irradiation feedback process is $F_{\text {irr }}=\alpha_{\text {irrad }} L_{\text {acc }} /\left(4 \pi a^{2}\right)$, where $a$ is the orbital separation, and $\alpha_{\text {irrad }}$ is considered as a free parameter.

As usual, we shall describe the rate of evaporation of the donor star $\dot{M}_{2, \text { evap }}$ with the simple prescription given by Stevens, Rees, \& Podsiadlowski (1992):

$\dot{M}_{2, \text { evap }}=-\frac{\alpha_{\text {evap }}}{2 v_{\text {esc }}^{2}} L_{\mathrm{PSR}}\left(\frac{R_{2}}{a}\right)^{2}$.

Here, $v_{\text {esc }}$ is the escape velocity from the donor star surface, $L_{\mathrm{PSR}}$ is the pulsar luminosity, and $\alpha_{\text {evap }}$ is a free parameter kept constant in our simulations.

In order to compute the luminosity of the pulsar $L_{\mathrm{PSR}}$ given by $L_{\mathrm{PSR}}=4 \pi^{2} I \dot{P}_{\mathrm{spin}} P_{\mathrm{spin}}^{-3}$ (where $I, P_{\mathrm{spin}}$, and $\dot{P}_{\mathrm{spin}}$ are the NS moment of inertia, spin period, and its derivative, respectively) we make the same assumptions, as in Chen et al. (2013): $I=10^{45} \mathrm{~g} \mathrm{~cm}^{2}$, initial $P_{\text {spin }}=3 \mathrm{msec}$, $\dot{P}_{\text {spin }}=10^{-20} s^{-1}$, and a braking index $n=3$. The evolution of the spin is computed considering the time since the pulsar emission starts out.

As usual, we assumed that the NS accretes mass with a rate given by $\dot{M}_{\mathrm{NS}}=\min \left[\beta \dot{M}_{2}, \dot{M}_{\text {Edd }}\right]$, where $\beta$ is the efficiency of accretion, and $\dot{M}_{\text {Edd }}$ is the critical Eddington rate that represents the upper value possible for a NS. The material lost from the system is assumed to carry the specific angular momentum of the NS.

\section{NUMERICAL RESULTS}

Let us ignore irradiation feedback and compute the evolution of a CBS with Solar composition 1,2 , and $3 M_{\odot}$ donor stars, together with a $1.4 M_{\odot}$ NS in tight orbits with initial periods $P=0.30,0.60$, and $0.60 \mathrm{~d}$. We shall consider different values for the parameter $\alpha_{\text {evap }}=0.010,0.030,0.075,0.100$, and 1.000 (from moderate to strong evaporation regimes) and assume that the pulsar starts to irradiate when the donor becomes fully convective, inhibiting further accretion onto the NS. This is essentially the exploration performed by Chen et al. (2013) for the case of a $1 M_{\odot}$ donor. The results are shown in Fig. 1 together with observational data 
on RBs presented below, in Table 2 and BWs taken from from Patruno's Catalogue1.

In our calculations, the donor stars with initial masses of 1,2 , and $3 M_{\odot}$ become fully convective when they have masses of $0.32 M_{\odot}, 0.14 M_{\odot}$, and $0.24 M_{\odot}$ at ages of $2.21,3.79$, and 2.12 Gyr, respectively. Since then on, evaporation leads to a departure from the predictions of "standard" CBS evolution.

Strong evaporation leads these systems to evolve to RB conditions, whereas moderate evaporation produce BWs. Within this scenario it seems difficult to populate the entire $\mathrm{RB}$ region indicated in the lower panel of Fig. 1 The reason is that this kind of models can only populate the region of masses lower than the one it has when becomes fully convective $\left(M_{2} \lesssim 0.32 M_{\odot}\right)$. This is appreciably lower than the high-mass edge of the $\mathrm{RB}$ region usually considered in the literature. Moreover, stellar models constructed this way lead to the occurrence of high filling factors $(F F \gtrsim 0.75)$ only shortly after detachment (less than 1 Gyr in the cases of 1 and $3 M_{\odot}$, and in an even more restricted time interval for the case of $2 M_{\odot}$ ). The tracks corresponding to $\alpha_{\text {evap }}=0.075$ can be considered as RB for a longer period. However, notice that they can populate a marginal portion of the plane shown in Fig. 1 and only for the cases of $1 M_{\odot}$ and $3 M_{\odot}$. For the rest of the tracks, the time spent by these systems as RBs with high filling factors is remarkably short. This can be seen in Fig. 2, where we show the evolution of the filling factors for the same calculations presented in Fig. 1

The models that consider irradiation feedback and do not impose any restriction to the accretion onto the NS component of the pair have been presented in Benvenuto, De Vito, \& Horvath $(2014 ; 2015 ; 2017)$. For appropriate initial conditions, these models undergo a sequence of cyclic mass transfer episodes. In each of these cycles, there is a short mass transfer episode followed by a long period in which the donor remains slightly detached from its Roche lobe, without shrinking back appreciably. Because of this reason we have called it as a "quasi-RLOF" and $F F \gtrsim 0.90$. For further details of this scenario we refer the reader to the above cited papers.

At this point we consider the possible scenario of irradiation feedback with radio ejection since first detachment. Let us consider the evolution of the same CBSs with an intermediate value for $\alpha_{\text {irrad }}=0.10$. This is enough for our purposes since previous models indicate that the onset of cyclic mass transfer does not depend strongly on this value (see, e.g. Benvenuto, De Vito, \& Horvath 2014; 2015). We assume that evaporation starts since the first detachment of the donor, and consider the same values of $\alpha_{\text {evap }}$ that we have used in the calculations presented in the previous paragraphs. Models constructed under these hypotheses are presented in Fig 3. The detachment and onset of evaporation of the models with 1,2 , and $3 M_{\odot}$ occurs when the donors have masses of $0.29,1.36$, and $1.22 M_{\odot}$ at the ages of $2.10,1.47$, and $1.10 \mathrm{Gyr}$, respectively. The model of $1 M_{\odot}$ behaves in a way similar to that in which irradiation feedback is ignored. However, remarkable differences are found for the cases of the models with 2 and $3 M_{\odot}$. In these cases detachment oc-
Table 1. The final masses of the NSs for the different conditions considered in this paper. We give the values for the initial masses of the donor stars and the orbital periods. In the third column we present the results for the case of strong evaporation without irradiation. In the fourth and fifth columns we give the masses for the cases of irradiation feedback with and without radio ejection, respectively.

\begin{tabular}{ccccc}
\hline \hline$M_{i} / M_{\odot}$ & $P_{i} / \mathrm{d}$ & $M_{N S} / M_{\odot}$ & $M_{N S} / M_{\odot}$ & $M_{N S} / M_{\odot}$ \\
\hline 1 & 0.3 & 1.74 & 1.75 & 1.85 \\
2 & 0.6 & 2.24 & 1.64 & 2.31 \\
3 & 0.6 & 2.03 & 1.54 & 2.13 \\
\hline \hline
\end{tabular}

curs when the models have a mass well above the high mass edge of the $\mathrm{RB}$ region. Therefore, when they evolve across it, they may behave as RBs. In this fashion the entire RB region can be populated. This is in sharp contrast with models that consider evaporation only. While these models have low filling factors in the case of heavy evaporation, they also may fill the entire Roche lobe if evaporation is slow.

Finally, we shall refer to the masses of the NSs found in our calculations (see Table 1). The masses found corresponding to the case of strongly evaporated models (column 3) are, generally speaking, higher that those found for the case of irradiation without radio ejection (column 4). On the other hand, we have made supplementary calculations in which we assumed the same initial condition (masses and orbital periods) and irradiation feedback, but without radio ejection. In this case we found slightly higher masses when comparing them with those of column 3 .

\section{OBSERVATIONAL DATA ON REDBACKS AND COMPARISON WITH THEORETICAL MODELS}

Some RBs have made transitions from accretion to pulsar state, or vice versa, confirming the model of recycled MSPs in CBSs. This group is know as transitional millisecond pulsars (tMSPs, PSR J1023+0038, PSR J1227-4853 and PSR J1824-2452I). On the other hand, there are some sources monitored because they have certain features that make them candidates to change its status (3FGL J2039.6-5618, 3FGL J0838.8-2829, 3FGL J0212.1+5320, 3FGL J0954.8-3948, 1FGL J0523.52529, 2FGL J0846.0+2820).

There are two particularly important states: the RLOF, where the donor star fills its Roche lobe $(F F=1)$, and the quasi-RLOF, where the donor is slightly smaller than its Roche lobe $(F F \approx 1)$.

In Table 2 we present the RBs, or candidates to RBs, known until today. In the first column, next to the name of the pulsar and in parentheses, we label with "S" the RBs in Table 1 of Smedley et al. (2015); with "F" that in the Freire's pulsars catalogue in globular clusters 2 , but not in Smedlev et al. (2015); with "A" the systems in the ATNF Pulsar Catalogue (Manchester, et al. 2005) with 

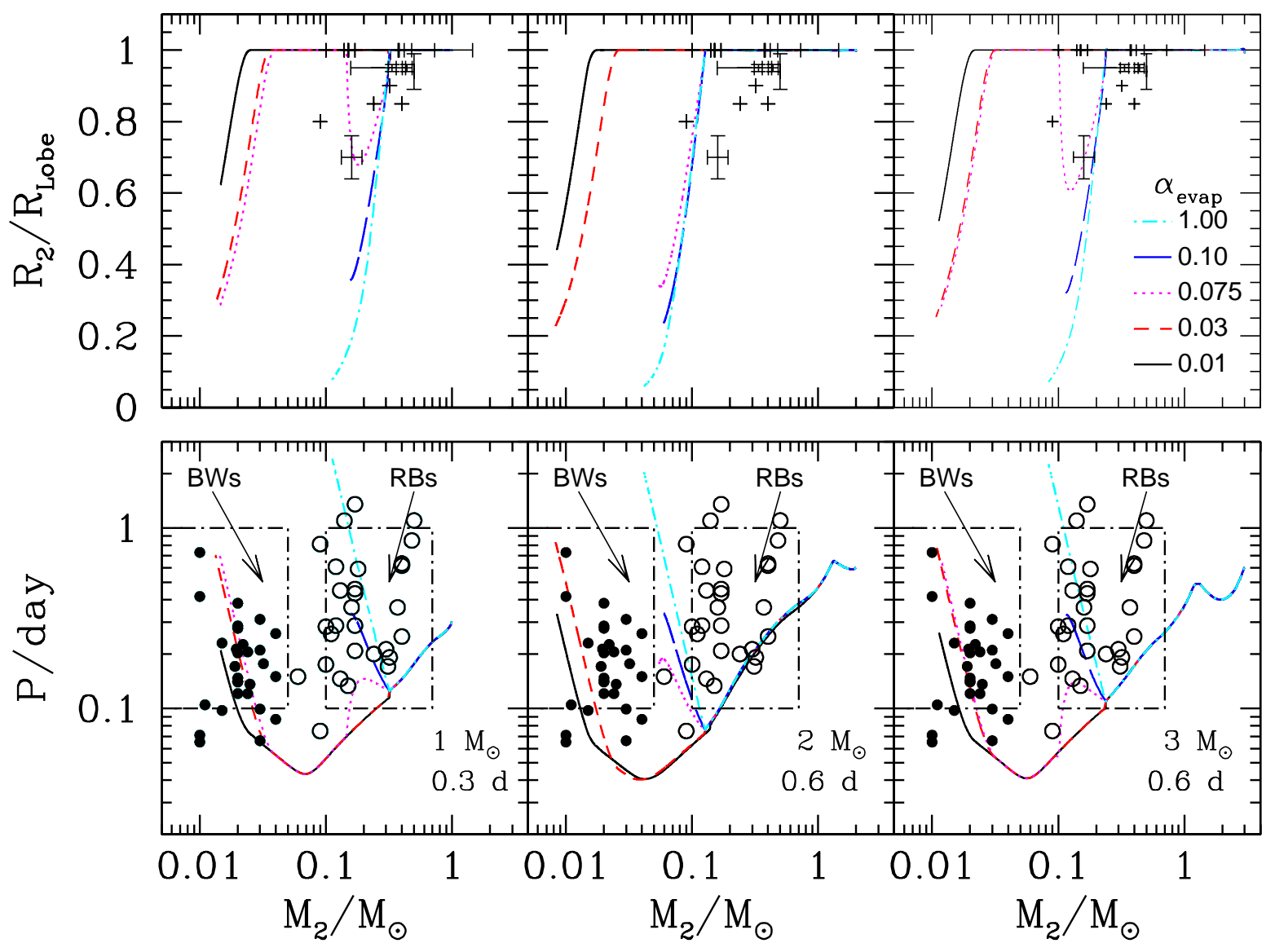

Figure 1. The evolution of CBSs with donor stars of 1,2 , and $3 M_{\odot}$ subject to different strengths of evaporation, switched on when they become fully convective. Lower panel shows the evolution of the orbital period as a function of donor mass. Strong evaporation regimes (corresponding to the highest considered values of $\alpha_{\text {evap }}$ ) allow to reach orbital periods corresponding to RBs. Upper panel shows the ratio of the donor radius to that of the equivalent Roche lobe (i.e., the filling factor $F F$ ) for the same set of evolutionary tracks. We have also plotted the $F F$ available in Table 2 Notice that strong evaporation allows reaching orbital periods in the whole range corresponding to RBs, but with low filling factors. Observed RBs listed in Table 2 are represented with open circles, whereas BWs are shown with solid circles.

$0.1 \mathrm{~d}<P<1 \mathrm{~d}, 0.1<M_{2} / M_{\odot}<0.7$ and main sequence or unknown companions; with "P" those in the Patruno's catalogue; with "L" in Linares (2018) and with "St" those in Strader et al. (2019). In the following columns we list the spin period of the pulsar, $P_{\mathrm{s}}$, the orbital period, $P$, the mass of the companion, $M_{2}$ (the best estimation of the mass, or, if it is not available, the minimum mas 4 ), and the filling factor FF. As we can see from the Table, most of the RBs do exhibit high filling factor values. In the cases where $F F$ has an estimation with $F F<1$, the minimum value is of $0.70(6)$ for PSR J1431-4715 (Strader et al. 2019) whereas the maximum value is of 0.92(6) for PSR J1306-40 Swihart, et al. 2019).

\footnotetext{
4 The minimum mass is computed considering a pulsar mass of $1.4 M_{\odot}$ and an inclination of $90^{\circ}$.
}

\section{DISCUSSION AND CONCLUSIONS}

In this work we have analysed different scenarios for the formation of binary RB pulsars and its relation with the filled fraction of the Roche lobe associated to the donor component of the pair. Moreover, we proposed that observations accurate enough of filling factors may be a powerful tool for discriminating among the mechanisms of $\mathrm{RB}$ formation proposed to date.

Strong evaporation scenarios have been presented by Chen et al. (2013) and Smedley et al. (2015). Chen et al. (2013) assumed the onset of evaporation since detachment of the donor when it becomes fully convective. Meanwhile Smedley et al. (2015) proposed that RBs are due to accretion induced collapse of a massive WD that detaches the donor from its lobe, allowing for the onset of evaporation. While close to the onset of evaporation the donor star has a 


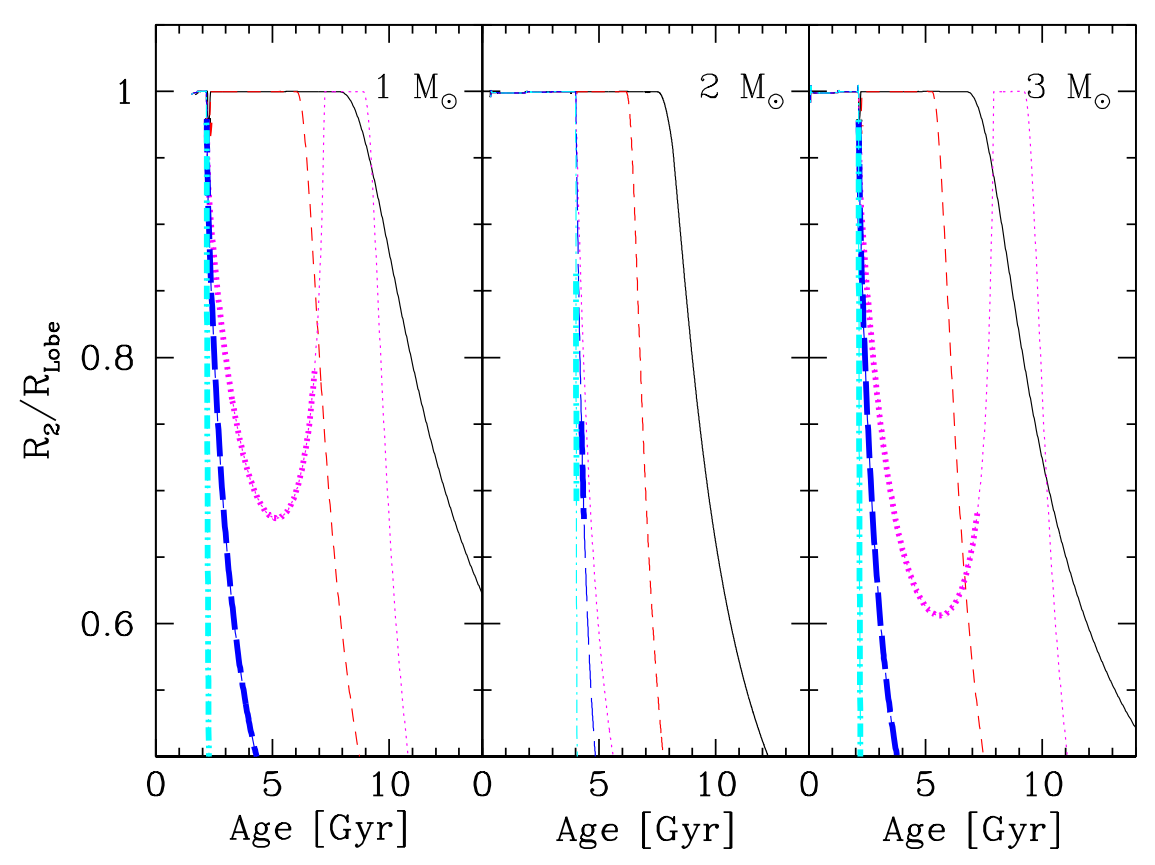

Figure 2. The filling factor $\left(F F=R_{2} / R_{\mathrm{RL}}\right)$ as a function of time for the evolutionary calculations presented in Fig. 1 Here we employ the same type and colour of lines as in that Figure. We denote the stages when the system fulfils the conditions on its mass and orbital period to be considered as a RB with heavy lines.

size comparable to its Roche lobe, in most cases soon after detachment both scenarios lead to low filling factors.

Our models with irradiation feedback (e.g., Benvenuto, De Vito, \& Horvath 2015) lead to high filling factors during the cyclic mass transfer evolutionary stages at which the system correspond to RB conditions (masses of the components, evolutionary stage of the donor star and orbital period). As the NS can accrete material, in this scenario we expect the occurrence of rather massive NSs. In this frame, we have analysed the possibility that after the first detachment induced by irradiation feedback, pulsar irradiation starts out and inhibits further accretion onto the NS; then, evaporation may drive the systems to become RBs. If evaporation is strong, it leads to the occurrence of low filling factors, similar to those predicted by the scenario proposed by Chen et al. (2013). However, it is also possible to find binary systems that evolve throughout the RBs region, essentially with full filled Roche lobes (i.e., filling factor one). In any case, radio ejection may represent a difficulty for the existence of some very massive NSs (see, e.g., Demorest et al. 2010, Cromartie, et al. 2019).

From the data presented in Table 2 there is some indication that the measured filling factors are generally high $(F F \gtrsim 0.75)$. This may, in principle be interpreted as an evidence in favour of models including irradiation feedback described above. In any case, we should remark that these non standard evolutionary paths are still rather uncertain. These uncertainties are encoded in the free parameters $\alpha_{\text {irr }}$ and $\alpha_{\text {evap }}$. We need a better understanding of the physics of irradiation and evaporation to get a better scenario of the process of RBs formation.

We would like to thank our anonymous referee for his/her valuable comments and suggestions that have helped us to largely improve the original version of this work.

J.E.H. has been supported by Fapesp (São Paulo, Brazil) through the grant 2013/26258-4 and CNPq, Brazil funding agencies.

\section{REFERENCES}

Archibald A. M., et al., 2009, Sci, 324, 1411

Bahramian A., et al., 2018, ApJ, 864, 28

Bates S. D., et al., 2015, MNRAS, 446, 4019

Bellm E. C., et al., 2016, ApJ, 816, 74

Benvenuto O. G., De Vito M. A., 2003, MNRAS, 342, 50

Benvenuto O. G., De Vito M. A., 2005, MNRAS, 362, 891

Benvenuto O. G., De Vito M. A., Horvath J. E., 2012, ApJ, 753, L33

Benvenuto O. G., De Vito M. A., Horvath J. E., 2014, ApJ, 786, L7

Benvenuto O. G., De Vito M. A., Horvath J. E., 2015, ApJ, 798, 44

Benvenuto O. G., De Vito M. A., Horvath J. E., 2017, A\&A, 598, A35

Boffin H. M. J., Hillen M., Berger J. P., Jorissen A., Blind N., Le Bouquin J. B., Mikolajewska J., Lazareff B., 2014, A\&A, 564, A1

Bogdanov S., Grindlay J. E., van den Berg M., 2005, ApJ, 630, 1029 

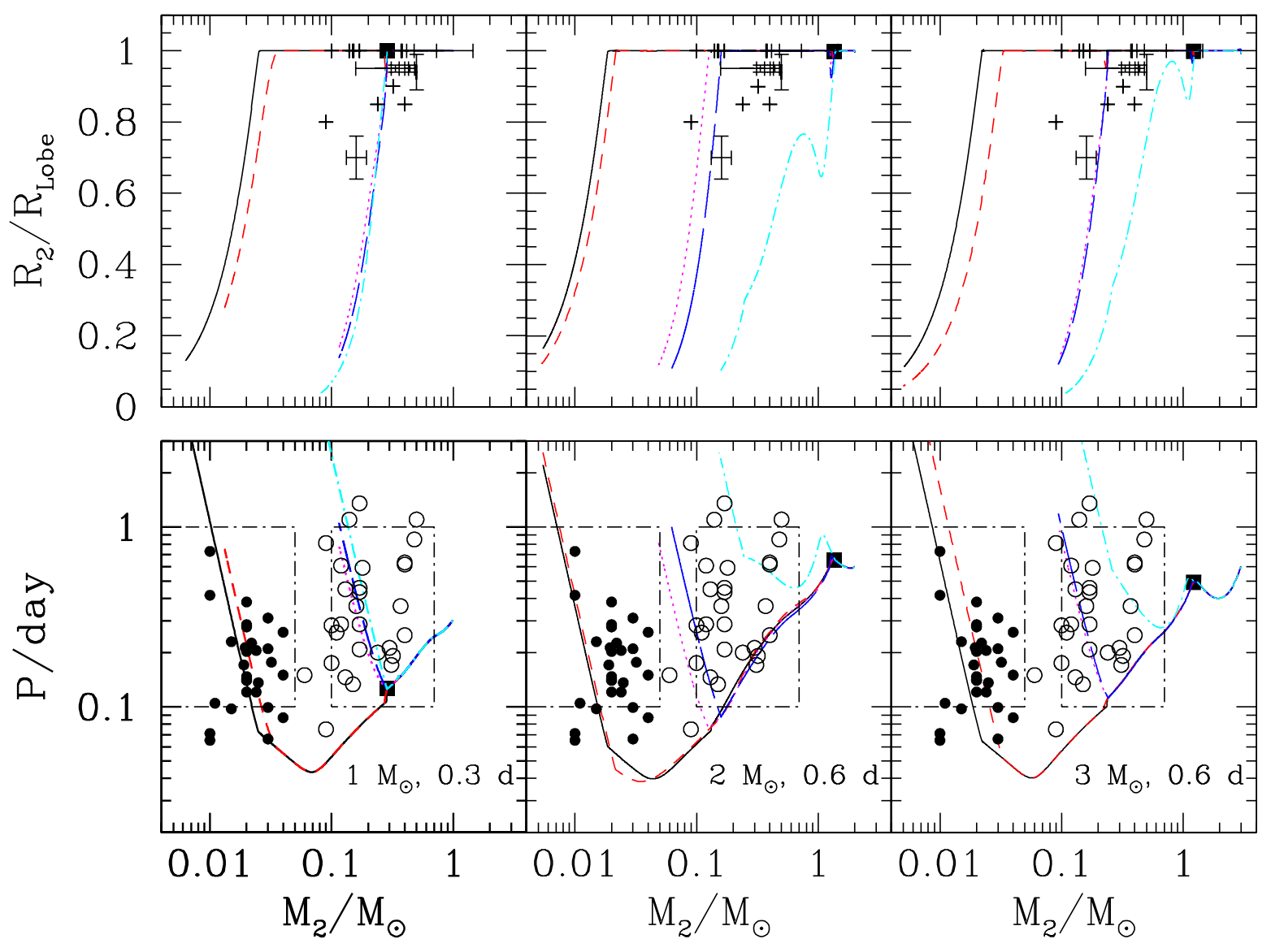

Figure 3. The evolution of the same CBSs presented in Fig. 1 but with evaporation starting at the first detachment of the donor due to irradiation feedback. Line types, observations, and regions for RBs and BWs are the same as in Fig. 1 Lower panels show the evolution of these systems, whereas upper panels depict the filling factors. Heavy squares on the tracks indicate the moment of the first detachment, when evaporation sets in. While for the case of $M_{2}=1 M_{\odot}$ detachment occurs approximately at the same conditions corresponding to the case of models without irradiation feedback, higher mass models detach when they are far more massive. Therefore, the entire RBs region can be populated in this way. As found for models without irradiation feedback, high evaporation rates lead to low filling factors, whereas moderate to low evaporation rates lead to (almost) completely filled lobes.

Büning A., Ritter H., 2004, A\&A, 423, 281

Burderi L., et al., 2001, ApJ, 560, L71 647

Burderi L., D'Antona F., di Salvo T., Riggio A., 2006, cosp, 36,1812

Chen H.-L., Chen X., Tauris T. M., Han Z., 2013, ApJ, 775,27

Cocozza G., Ferraro F. R., Possenti A., Beccari G., Lanzoni B., Ransom S., Rood R. T., D'Amico N., 2008, ApJ, 679, L105

Crawford F., et al., 2013, ApJ, 776, 20

Cromartie H. T., et al., 2019, NatAs.tmp, 439

D'Amico N., Lyne A. G., Manchester R. N., Possenti A., Camilo F., 2001, ApJ, 548, L171

Demorest P. B., Pennucci T., Ransom S. M., Roberts M. S. E., Hessels J. W. T., 2010, Natur, 467, 1081

Deneva J. S., et al., 2016, ApJ, 823, 105

Eggleton P. P., 1983, ApJ, 268, 368
Freire P. C. C., Ransom S. M., Bégin S., Stairs I. H., Hessels J. W. T., Frey L. H., Camilo F., 2008, ApJ, 675, 670

Hessels J. W. T., Ransom S. M., Stairs I. H., Kaspi V. M., Freire P. C. C., 2007, ApJ, 670, 363

Janssen T., van Kerkwijk M. H., 2005, A\&A, 439, 433

Kaplan D. L., et al., 2012, ApJ, 753, 174

Kaplan D. L., Bhalerao V. B., van Kerkwijk M. H., Koester D., Kulkarni S. R., Stovall K., 2013, ApJ, 765, 158

Li M., Halpern J. P., Thorstensen J. R., 2014, ApJ, 795, 115

Linares M., 2018, MNRAS, 473, L50

Linares M., Shahbaz T., Casares J., 2018, ApJ, 859, 54

McConnell O., Callanan P. J., Kennedy M., Hurley D., Garnavich P., Menzies J., 2015, MNRAS, 451, 3468

Manchester R. N., Hobbs G. B., Teoh A., Hobbs M., 2005, AJ, 129, 1993

Malkov O. Y., 2003, A\&A, 402, 1055 
Table 2. Relevant observational data for Redback Pulsars. We tabulate the name of the pulsar and in parentheses the source of data: "S" from Smedley et al. (2015); "F" from Freire's catalogue of pulsars in globular clusters; "A" from the ATNF Pulsar Catalogue (Manchester, et al. 2005) with $0.1 \mathrm{~d}<P<1 \mathrm{~d}, 0.1<M_{2} / M_{\odot}<0.7$ and main sequence or unknown companions; "P" from Patruno's catalogue; "L" from Linares (2018) and "St" from Strader et al. (2019). In the following columns we list the spin period of the pulsar $P_{s}$, the orbital period $P$, the mass of the companion $M_{2}$ (see main text), and the filling factor $F F$. In the last column we have included the main references.

\begin{tabular}{|c|c|c|c|c|c|}
\hline PSR & $P_{s}[\mathrm{~ms}]$ & $P[\mathrm{~h}]$ & $M_{2}\left[M_{\odot}\right]$ & $F F$ & Reference \\
\hline J0024-7204V (F) & 4.81 & 5.1 & $0.30-1.17$ & $\mathrm{RLOF}^{1}$ & Ridolfi et al. (2016) \\
\hline J0024-7204W (P) & 2.35 & 3.2 & 0.15 & $\mathrm{RLOF}^{1}$ & Bogdanov, Grindlav, \& van den Berg (2005); Ridolfi et al. (2016) \\
\hline $\mathrm{J} 1023+0038(\mathrm{~S})$ & 1.69 & 4.8 & 0.24 & $0.85^{2}$ & Archibald et al. (2009); McConnell et al. (2015) \\
\hline $\mathrm{J} 1048+2339(\mathrm{~A})$ & 4.66 & 6.0 & $\sim 0.4$ & 0.85 & Deneva et al. (2016); Yap et al. (2019) \\
\hline $\mathrm{J} 1227-4853(\mathrm{~A})$ & 1.69 & 6.9 & $0.17-0.46$ & quasi-RLOF ${ }^{2}$ & (Rov et al. 2015) \\
\hline $\mathrm{J} 1306-40(\mathrm{~L})$ & 2.20 & 26.33 & $\sim 0.5$ & $0.92(6)$ & Linares (2018) \\
\hline $\mathrm{J} 1431-4715(\mathrm{~A})$ & 2.01 & 10.8 & $0.13-0.19$ & $0.70(6)$ & Bates et al. (2015); Strader et al. (2019) ${ }^{*}$ \\
\hline J1622-0315 (St) & 3.85 & 3.9 & $\geqslant 0.10$ & - & Strader et al. $(2019)^{*}$ \\
\hline $\mathrm{J} 1628-3205(\mathrm{~S})$ & 3.21 & 5.0 & $0.17-0.24$ & quasi-RLOF & Li, Halpern, \& Thorstensen (2014); Strader et al. $(2019)^{*}$ \\
\hline $\mathrm{J} 1641+3627 \mathrm{D}(\mathrm{A})$ & 3.12 & 14.2 & 0.18 & - & Hessels et al. (2007) \\
\hline J1701-3006B (S) & 3.59 & 3.5 & $0.13-0.41$ & RLOF & Cocozza et al. (2008) \\
\hline J1721-1936 (A) & 1000.00 & 6.2 & $0.11-0.27$ & quasi-RLOF & Janssen \& van Kerkwiik (2005) \\
\hline $\mathrm{J} 1723-2837(\mathrm{~S})$ & 1.86 & 14.8 & $0.40-0.70$ & $\sim 1$ & Crawford et al. (2013); van Staden \& Antoniadis (2016) \\
\hline J1740-5340A (S) & 3.65 & 32.4 & $0.14-0.38$ & $\sim 0.95$ & D'Amico et al. (2001); Orosz \& van Kerkwiik (2003) \\
\hline J1748-2021D (S) & 13.50 & 6.9 & 0.12 & - & Freire et al. (2008) \\
\hline J1748-2446A (S) & 11.56 & 1.8 & 0.09 & $\sim 0.8$ & Nice \& Thorsett (1992) \\
\hline J1748-2446ad (S) & 1.40 & 26.3 & 0.14 & RLOF & Burderi et al. (2006) \\
\hline J1748-2446ai (F) & 21.23 & 20.4 & 0.48 & - & Freire's pulsars catalogue \\
\hline $\mathrm{J} 1748-2446 \mathrm{P}(\mathrm{S})$ & 1.73 & 8.7 & 0.38 & $\mathrm{RLOF}^{1}$ & Ransom et al. (2005) \\
\hline $\mathrm{J} 1816+4510(\mathrm{P})$ & 3.19 & 8.7 & $\leqslant 0.19(5)$ & $<1$ & Kaplan et al. (2013) \\
\hline J1824-2452H (S) & 4.62 & 10.4 & 0.17 & RLOF & Pallanca et al. (2010) \\
\hline J1824-2452I (S) & 3.93 & 11.0 & 0.17 & quasi-RLOF ${ }^{2}$ & Papitto et al. (2013) \\
\hline $\mathrm{J} 1905+0154 \mathrm{~A}(\mathrm{~A})$ & 3.19 & 19.5 & 0.09 & - & Hessels et al. (2007) \\
\hline $\mathrm{J} 1906+0055(\mathrm{~A})$ & 2.79 & 14.6 & 0.12 & - & Stovall et al. (2016) \\
\hline $\mathrm{J} 1908+2105(\mathrm{P})$ & 2.56 & 3.6 & 0.06 & - & Strader et al. $(2019)^{*}$ \\
\hline $\mathrm{J} 1957+2516(\mathrm{St})$ & 4.00 & 6.8 & 0.10 & - & Stovall et al. (2016) \\
\hline $\mathrm{J} 2129-0429(\mathrm{~S})$ & 7.62 & 15.2 & $0.44(4)$ & $0.95(1)$ & Bellm et al. (2016) \\
\hline J2140-2310A (S) & 11.02 & 4.2 & 0.1 & $\mathrm{RLOF}^{3}$ & Ransom et al. (2004) \\
\hline $\mathrm{J} 2215+5135(\mathrm{~S})$ & 2.61 & 4.1 & $0.33_{-0.02}^{+0.03}$ & $0.95(1)$ & Linares, Shahbaz, \& Casares (2018) \\
\hline J2339-0533 (A) & 2.88 & 4.6 & 0.32 & $\sim 0.90$ & Pletsch \& Clark (2015) \\
\hline
\end{tabular}

${ }^{1}$ From eclipses.

2 Transitional.

${ }^{3}$ A significant amount of the material in the eclipsing region is outside the companion's Roche lobe.

* See also references therein.

Nice D. J., Thorsett S. E., 1992, ApJ, 397, 249

Orosz J. A., van Kerkwijk M. H., 2003, A\&A, 397, 237

Pallanca C., et al., 2010, ApJ, 725, 1165

Papitto A., et al., 2013, Natur, 501, 517

Phinney E. S., Evans C. R., Blandford R. D., Kulkarni

S. R., 1988, Natur, 333, 832

Pletsch H. J., Clark C. J., 2015, ApJ, 807, 18

Podsiadlowski P., Rappaport S., Pfahl E. D., 2002, ApJ, 565,1107

Ransom S. M., Stairs I. H., Backer D. C., Greenhill L. J., Bassa C. G., Hessels J. W. T., Kaspi V. M., 2004, ApJ, 604, 328

Ransom S. M., Hessels J. W. T., Stairs I. H., Freire P. C. C., Camilo F., Kaspi V. M., Kaplan D. L., 2005, Sci, 307, 892

Ridolfi A., et al., 2016, MNRAS, 462, 2918

Roberts M. S. E., 2013, IAUS, 291, 127

Roy J., et al., 2015, ApJ, 800, L12

Smedley S. L., Tout C. A., Ferrario L., Wickramasinghe D. T., 2015, MNRAS, 446, 2540

Stevens I. R., Rees M. J., Podsiadlowski P., 1992, MNRAS,
$254,19 \mathrm{P}$

Stovall K., et al., 2016, ApJ, 833, 192

Strader J., et al., 2019, ApJ, 872, 42

Swihart S. J., Strader J., Chomiuk L., Shishkovsky L., 2019, ApJ, 876, 8

van Staden A. D., Antoniadis J., 2016, ApJ, 833, L12

Yap Y. X., Li K. L., Kong A. K. H., Takata J., Lee J., Hui C. Y., 2019, A\&A, 621, L9 